\title{
Torus knots and Dunwoody manifolds
}

\author{
Hüseyin Aydin Inci Gültekyn Michele Mulazzani
}

\begin{abstract}
We obtain an explicit representation, as Dunwoody manifolds, of all cyclic branched coverings of torus knots of type $(p, m p \pm 1)$, with $p>1$ and $m>0$.

Mathematics Subject Classification 2000: Primary 57M05, 20F38; Secondary 57M12, 57M25.

Keywords: torus knots, Heegaard splittings, Dunwoody manifolds.
\end{abstract}

\section{Introduction}

Many interesting examples of cyclic branched coverings of knots in $\mathbf{S}^{3}$ admitting cyclic presentations for the fundamental groups have recently been found (see 4, 5, 17, 10, 11, 12, 16]). In order to investigate these relations, M. J. Dunwoody introduced in [5] a class of 3-manifolds, depending on six integer parameters, with cyclically presented fundamental groups. It has been shown in [6] that all these manifolds turn out to be strongly-cyclic branched coverings of $(1,1)$-knots in lens spaces (possibly $\mathbf{S}^{3}$ ). Moreover, the explicit Dunwoody representation for all cyclic branched coverings of 2-bridge knots has been obtained.

In this paper we give a similar result for a wide class of torus knots, which are, together with 2-bridge knots, the most important examples of $(1,1)$-knots in $\mathbf{S}^{3}$. The Dunwoody parameters are obtained for all cyclic branched coverings of torus knots of type $(p, m p \pm 1)$, with $p>1$ and $m>0$, thus including all torus knots with bridge number $\leq 4$. These manifolds have been considered, from a different point of view, in [1] and [4].

We refer to 9, 15, for details on knot theory and cyclic branched coverings of knots and to [8] for details on cyclic presentation of groups. 


\section{2 (1,1)-knots and Dunwoody manifolds}

A knot $K$ in a 3-manifold $N^{3}$ is called a $(1,1)$-knot if there exists a Heegaard splitting of genus one

$$
\left(N^{3}, K\right)=(T, A) \cup_{\varphi}\left(T^{\prime}, A^{\prime}\right),
$$

where $T$ and $T^{\prime}$ are solid tori, $A \subset T$ and $A^{\prime} \subset T^{\prime}$ are properly embedded trivial arcs, and $\varphi:\left(\partial T^{\prime}, \partial A^{\prime}\right) \rightarrow(\partial T, \partial A)$ is an attaching homeomorphism (see Figure (1). Obviously, $N^{3}$ turns out to be a lens space $L(p, q)$ (including $\left.\mathbf{S}^{3}=L(1,0)\right)$.
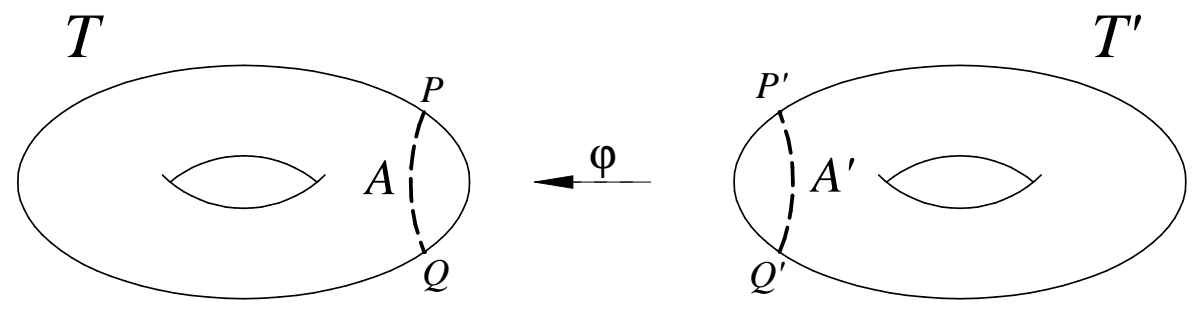

Figure 1: A (1,1)-decomposition.

It is well known that the family of $(1,1)$-knots contains all torus knots and all two-bridge knots in $\mathbf{S}^{3}$. Several topological properties of $(1,1)$-knots have recently been investigated (see references in [2]).

An algebraic representation of $(1,1)$-knots has been developed in [1] and [2], where it is shown that there is a natural surjective map

$$
\psi \in P M C G_{2}(\partial T) \mapsto K_{\psi} \in \mathcal{K}_{1,1}
$$

from the pure mapping class group of the twice punctured torus $P M C G_{2}(\partial T)$ to the class $\mathcal{K}_{1,1}$ of all $(1,1)$-knots.

The fundamental group of the exterior of a $(1,1)$-knot $K_{\psi}$ can be explicitly obtained using its representation $\psi$. Let $\alpha, \beta, \gamma \subset \partial T$ be the loops depicted in Figure 2] They represent a set of free generators for $\pi_{1}(\partial T-\partial A, *)$, while $\alpha$ and $\gamma$ freely generate $\pi_{1}(T-A, *)$. A straightforward application of Seifert-Van Kampen theorem gives:

Lemma 1 [1] The fundamental group of the exterior of a $(1,1)$-knot $K_{\psi} \subset$ $L(p, q)$ admits the presentation

$$
\pi_{1}\left(L(p, q)-K_{\psi}, *\right)=\langle\alpha, \gamma \mid r(\alpha, \gamma)\rangle,
$$


where $r(\alpha, \gamma)$ is the homotopy class of $i(\psi(\beta))$, being $i:(\partial T, \partial A) \rightarrow(T, A)$ the inclusion map.

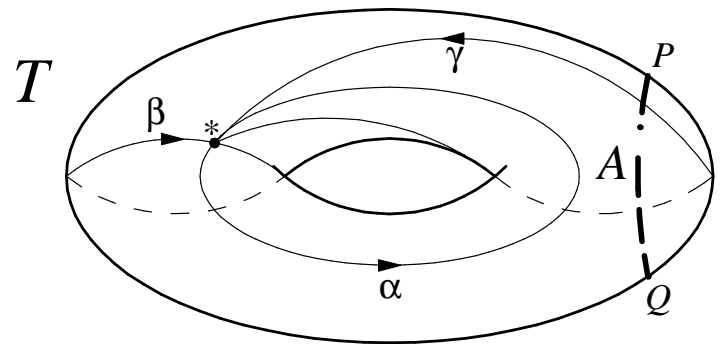

Figure 2:

As a consequence, all $(1,1)$-knots in $\mathbf{S}^{3}$ are prime [14], and can therefore be classified, up to mirror image, by their fundamental groups (see [9, p. 76]). Many results concerning the connections between cyclically presented groups and strongly-cyclic branched coverings of $(1,1)$-knots (see definition in [1]) have been obtained. It has been proved in [13] that every $n$-fold stronglycyclic branched covering of a $(1,1)$-knot admits a Heegaard diagram of genus $n$ which encodes a cyclic presentation for the fundamental group. This result has been improved in [1], where a constructive algorithm which explicitly gives the cyclic presentations is obtained.

The family of Dunwoody manifolds has been introduced in [5] by a class of trivalent regular planar graphs (called Dunwoody diagrams) with cyclic symmetry, depending on six integers $a, b, c, n, r, s$, such that $n>0, a, b, c \geq 0$ and $a+b+c>0$. For certain values of the parameters, called admissible, the Dunwoody diagrams $D(a, b, c, n, r, s)$ turn out to be Heegaard diagrams, so defining a wide class of closed, orientable 3-manifolds $M(a, b, c, n, r, s)$ with cyclically presented fundamental groups.

More precisely, an admissible Dunwoody diagram $D(a, b, c, n, r, s)$ is an open Heegaard diagram of genus $n$ (see Figure 31), which contains $n$ upper cycles $C_{1}^{\prime}, \ldots, C_{n}^{\prime}$, and $n$ lower cycles $C_{1}^{\prime \prime}, \ldots, C_{n}^{\prime \prime}$, each having $d=2 a+b+c$ vertices. For every $i=1, \ldots, n$, the cycle $C_{i}^{\prime}$ (resp. $C_{i}^{\prime \prime}$ ) is connected to the cycle $C_{i+1}^{\prime}$ (resp. $C_{i+1}^{\prime \prime}$ ) by a parallel arcs, to the cycle $C_{i}^{\prime \prime}$ by $c$ parallel arcs and to the cycle $C_{i+1}^{\prime \prime}$ by $b$ parallel arcs. We denote by $\mathcal{B}$ the set of all these arcs. The cycle $C_{i}^{\prime}$ is glued to the cycle $C_{i-s}^{\prime \prime}(\operatorname{subscripts} \bmod n)$ so that 


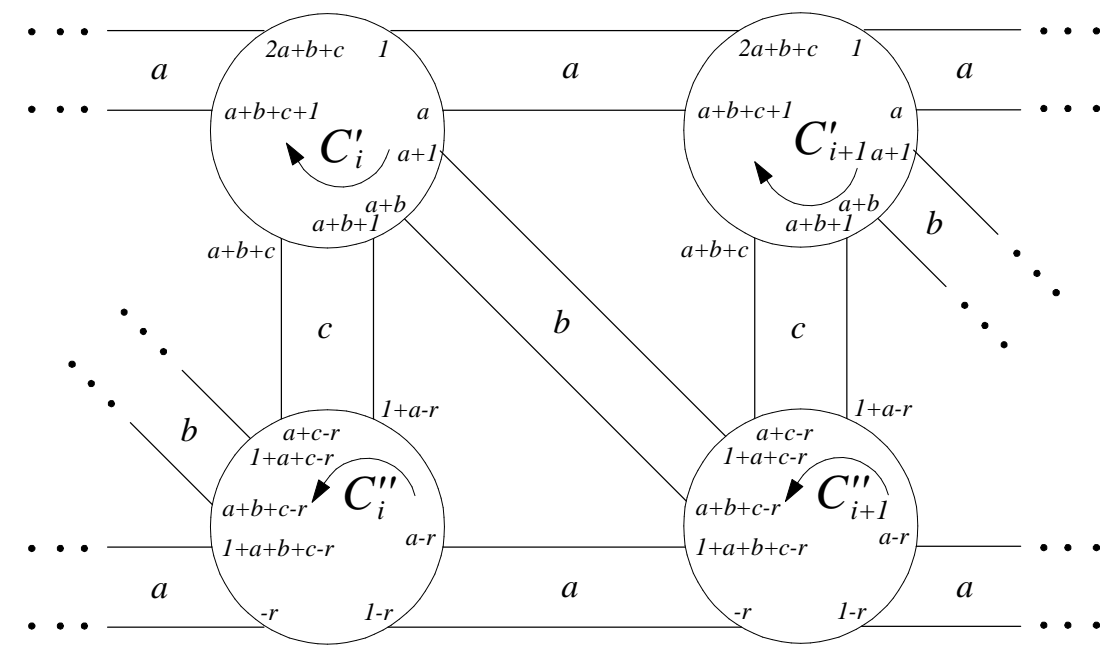

Figure 3: A Dunwoody diagram.

equally labelled vertices are identified together. Observe that the parameters $r$ and $s$ can be considered mod $d$ and $n$ respectively. Since the identification rule and the diagram are invariant with respect to a cyclic action of order $n$, the Dunwoody manifolds admit a cyclic symmetry of order $n$. Obviously, the Dunwoody manifold $M(a, b, c, 1, r, 0)$ is homeomorphic to a lens space (possibly $\mathbf{S}^{3}$ ), since it admits a Heegaard splitting of genus one.

A characterization of all Dunwoody manifolds as strongly-cyclic branched coverings of $(1,1)$-knots is given by the following:

Proposition 2 [6] The Dunwoody manifold $M(a, b, c, n, r, s)$ is the $n$-fold strongly-cyclic covering of the lens space $M(a, b, c, 1, r, 0)$ (possibly $\mathbf{S}^{3}$ ), branched over a $(1,1)$-knot $K(a, b, c, r)$ only depending on the integers $a, b, c, r$.

The converse of this result is also true, as proved in [3]. So the class of the Dunwoody manifolds coincides with the class of the strongly-cyclic branched coverings of $(1,1)$-knots.

The $(1,1)$-knots $K(a, b, c, r)$ occurring in Proposition 2 admit a natural $(1,1)$-decomposition $(T, A) \cup_{\varphi}\left(T^{\prime}, A^{\prime}\right)$ depicted in Figure 4, where the arcs of $\mathcal{B}$ constitute the curve $\varphi\left(\beta^{\prime}\right)=\psi(\beta), \psi$ being the element of $P M C G_{2}(\partial T)$ correspondent to $\varphi$. The fundamental group of the exterior of $K(a, b, c, r)$ can 


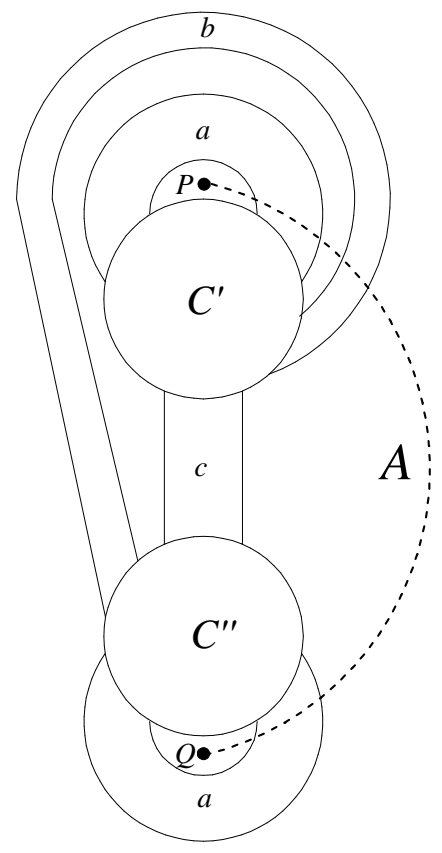

Figure 4: $D(a, b, c, 1, r, 0)$

be directly read in the Dunwoody diagram of $D(a, b, c, 1, r, 0)$. The relation $r(\alpha, \gamma)$ of the presentation of Lemma 1 is obtained by walking along the arcs of $\mathcal{B}$, following a fixed orientation: associate to each arc a word in $\alpha$ and $\gamma$ representing its homotopy class in the fundamental group of $T-A$ (see Figure 5), where a properly embedded disk with boundary $C$ is considered squeezed to the base point $*$.

\section{Main results}

An interesting problem is to find the Dunwoody parameters of the cyclic branched coverings of important classes of $(1,1)$-knots, in particular when the knot lies in $\mathbf{S}^{3}$. This type of result has been obtained in [6, Theorem 8] for all 2-bridge knots.

Now we obtain a similar result for the torus $\operatorname{knots} \mathbf{t}(p, m p \pm 1)$, with $m>0$ and $p>1$. 

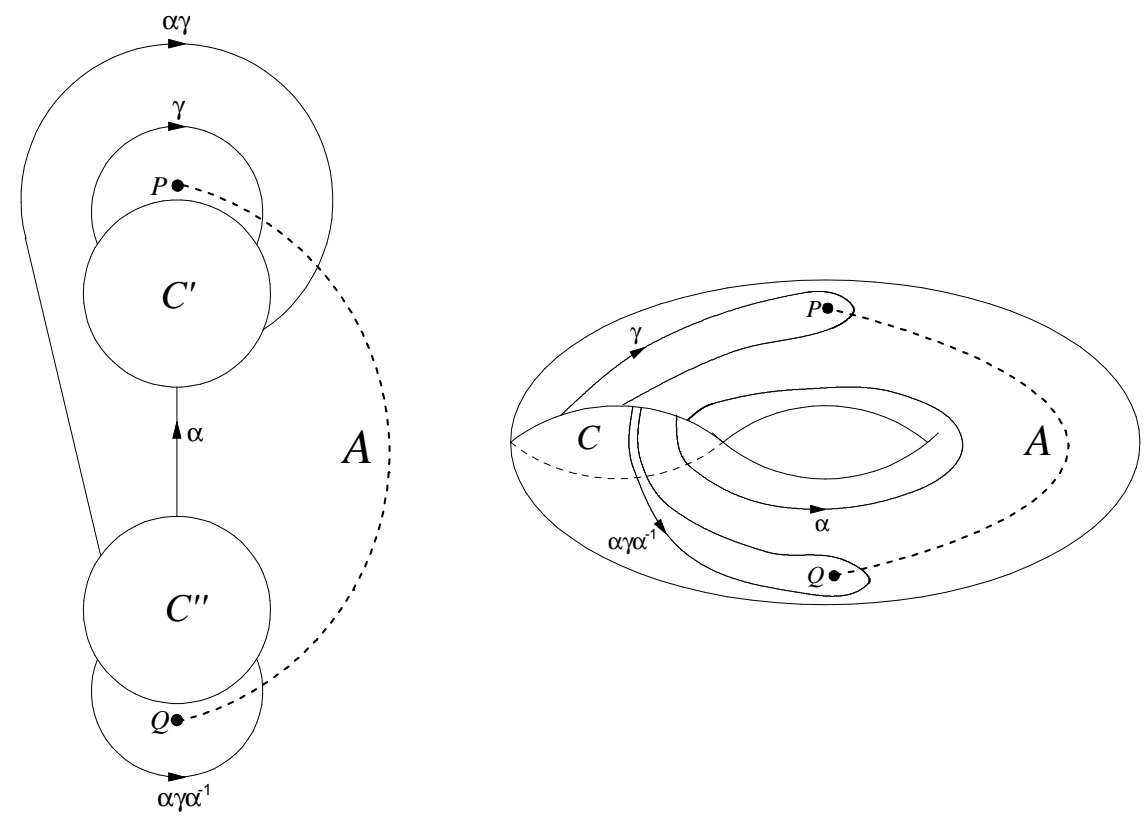

Figure 5:

Proposition 3 1. $K(1, p-2,2 m p-2 m-p+1, p)$ is the torus knot $\mathbf{t}(p, m p+1)$, for all $m>0$ and $p>1$;

2. $K(1, p-2,2 m p-2 m-p-1,-3 p+4)$ is the torus $k n o t \mathbf{t}(p, m p-1)$, for all $m>1$ and $p>1$.

Proof. 1. Let $K=K(1, p-2,2 m p-2 m-p+1, p)$. Figure [ depicts the Dunwoody diagram $D=D(1, p-2,2 m p-2 m-p+1,1, p, 0)$. The number of vertices of each cycle is $d=2 m(p-1)+1$. Starting from the vertex of $C^{\prime \prime}$ with label $d$, we pass along all the arcs of $\mathcal{B}$ with the described orientation, obtaining the word $w=\alpha^{m(p-1)} \alpha \gamma^{-1} \alpha^{-1}\left(\alpha^{-(m-1)} \gamma^{-1} \alpha^{-1}\right)^{p-2} \alpha^{-(m-1)} \gamma^{-1}$. Therefore, since the conditions of [6, Corollary 4] are satisfied, $D$ is admissible. Moreover, the fundamental group of $M(1, p-2,2 m p-2 m-p+1,1, p, 0)$ is $\langle\alpha, \gamma \mid w, \gamma\rangle$, which is trivial. So $M(1, p-2,(p-1)(2 m-1), 1, p, 0) \cong \mathbf{S}^{3}$. Moreover, $\pi_{1}\left(\mathbf{S}^{3}-K\right)=\langle\alpha, \gamma \mid w\rangle$. Since $w=\alpha^{m(p-1)+1}\left(\gamma^{-1} \alpha^{-m}\right)^{p-1} \gamma^{-1}=$ $\alpha^{-m} \alpha^{m p+1}\left(\gamma^{-1} \alpha^{-m}\right)^{p} \alpha^{m}$, we have $\pi_{1}\left(\mathbf{S}^{3}-K\right) \cong\left\langle\alpha, \gamma \mid w^{\prime}\right\rangle$, with $w^{\prime}=$ $\alpha^{m p+1}\left(\gamma^{-1} \alpha^{-m}\right)^{p}$. Obviously this group is isomorphic to the group $\langle x, y|$ 
$\left.x^{m p+1} y^{-p}\right\rangle$, which is the group of the torus knot $\mathbf{t}(p, m p+1)$. As a consequence $K$ is precisely $\mathbf{t}(p, m p+1)$.

2. The proof is analogous to the previous one. Let $K=K(1, p-2,2 m p-$ $2 m-p-1,-3 p+4)$. Figure 7 depicts the Dunwoody diagram $D=D(1, p-$ $2,2 m p-2 m-p-1,1,-3 p+4,0)$. The number of vertices of each cycle is $d=2 m(p-1)-1$ (note that the labelling is considered mod $d$ ). Starting from the vertex of $C^{\prime \prime}$ with label $d$, we pass along all the $\operatorname{arcs}$ of $\mathcal{B}$, obtaining the word $w=\alpha^{m-1}\left(\alpha^{m}\right)^{p-3} \alpha^{m-1} \alpha \gamma^{-1} \alpha^{-1} \alpha^{-(m-1)}\left(\gamma^{-1} \alpha^{-1} \alpha^{-(m-1)}\right)^{p-2} \gamma^{-1}$. So $D$ is admissible. Moreover, $M(1, p-2,2 m p-2 m-p-1,1,-3 p+4,0)$ is homeomorphic to $\mathbf{S}^{3}$, since its fundamental group is trivial. Since $w=$ $\alpha^{m(p-1)-1}\left(\gamma^{-1} \alpha^{-m}\right)^{p-1} \gamma^{-1}=\alpha^{m p-1}\left(\alpha^{-m} \gamma^{-1}\right)^{p}$, the group $\pi_{1}\left(\mathbf{S}^{3}-K\right) \cong\langle\alpha, \gamma|$ $w\rangle$ is isomorphic to the group $\left\langle x, y \mid x^{m p-1} y^{-p}\right\rangle$. Therefore, $K$ is the torus $\operatorname{knot} \mathbf{t}(p, m p-1)$.

Corollary 4 1. For all $m>0$ and $p>1$, the $n$-fold cyclic branched covering of the torus knot $\mathbf{t}(p, m p+1)$ is the Dunwoody manifold $M(1, p-2,2 m p-2 m-p+1, n, p, p)$;

2. for all $m>1$ and $p>1$, the $n$-fold cyclic branched covering of the torus knot $\mathbf{t}(p, m p-1)$ is the Dunwoody manifold $M(1, p-2,2 m p-2 m-p-1, n,-3 p+4,-p)$.

Proof. From Proposition 2, we only need to find the sixth parameter $s$ of the 6-tuples. The relation $q_{\sigma}+s p_{\sigma} \equiv 0 \bmod n$ must be satisfied for all $n$, where $p_{\sigma}$ is the number of arcs of $\mathcal{B}$ oriented from $C^{\prime}$ to $C^{\prime \prime}$ minus the number of the ones oriented from the $C^{\prime \prime}$ to $C^{\prime}$ and $q_{\sigma}$ is the number of arcs of $\mathcal{B}$ oriented from the right to the left minus the number of the ones oriented from the left to the right in the Dunwoody diagram (see [6, p. 385]). In other words, $-p_{\sigma}$ is the total exponent of $\alpha$ in $w$ and $-q_{\sigma}$ is the total exponent of $\gamma$ in $w$. In the first case $q_{\sigma}=p$ and $p_{\sigma}=-1$; therefore $s=p$. In the second case $q_{\sigma}=p$ and $p_{\sigma}=1$; therefore $s=-p$.

An extension of the previous results to the whole class of torus knots by using the same proof technique seems to be very complicated, although some partial results have been obtained. A possible alternative method could be to work by induction on the number of steps of the Euclidean division algorithm needed to obtain the greatest common division of the parameters of the torus knot. Following this approach, the results of this article could represent the first inductive step. 


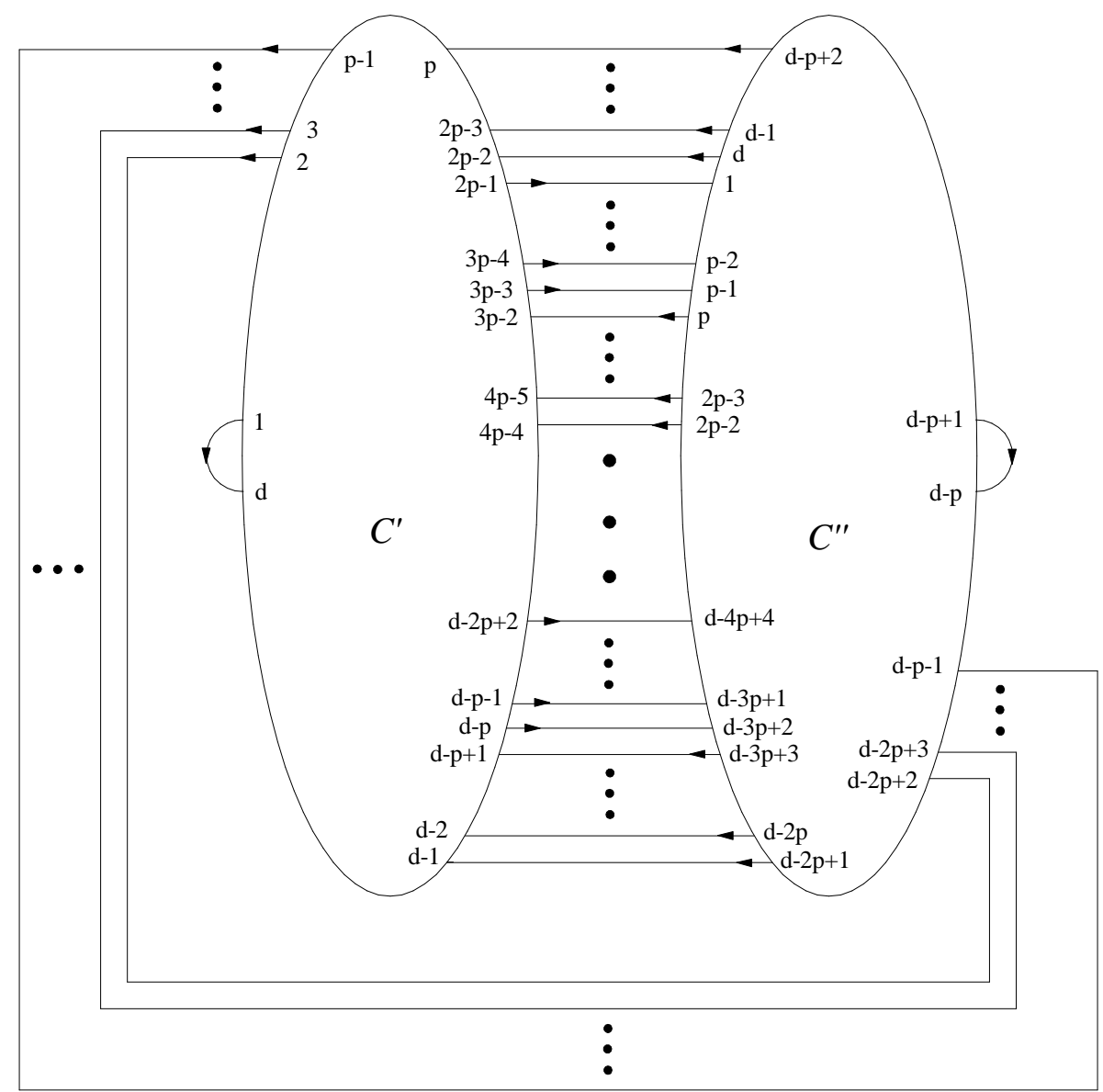

Figure 6: $D(1, p-2,2 m p-2 m-p+1,1, p, 0)$. 


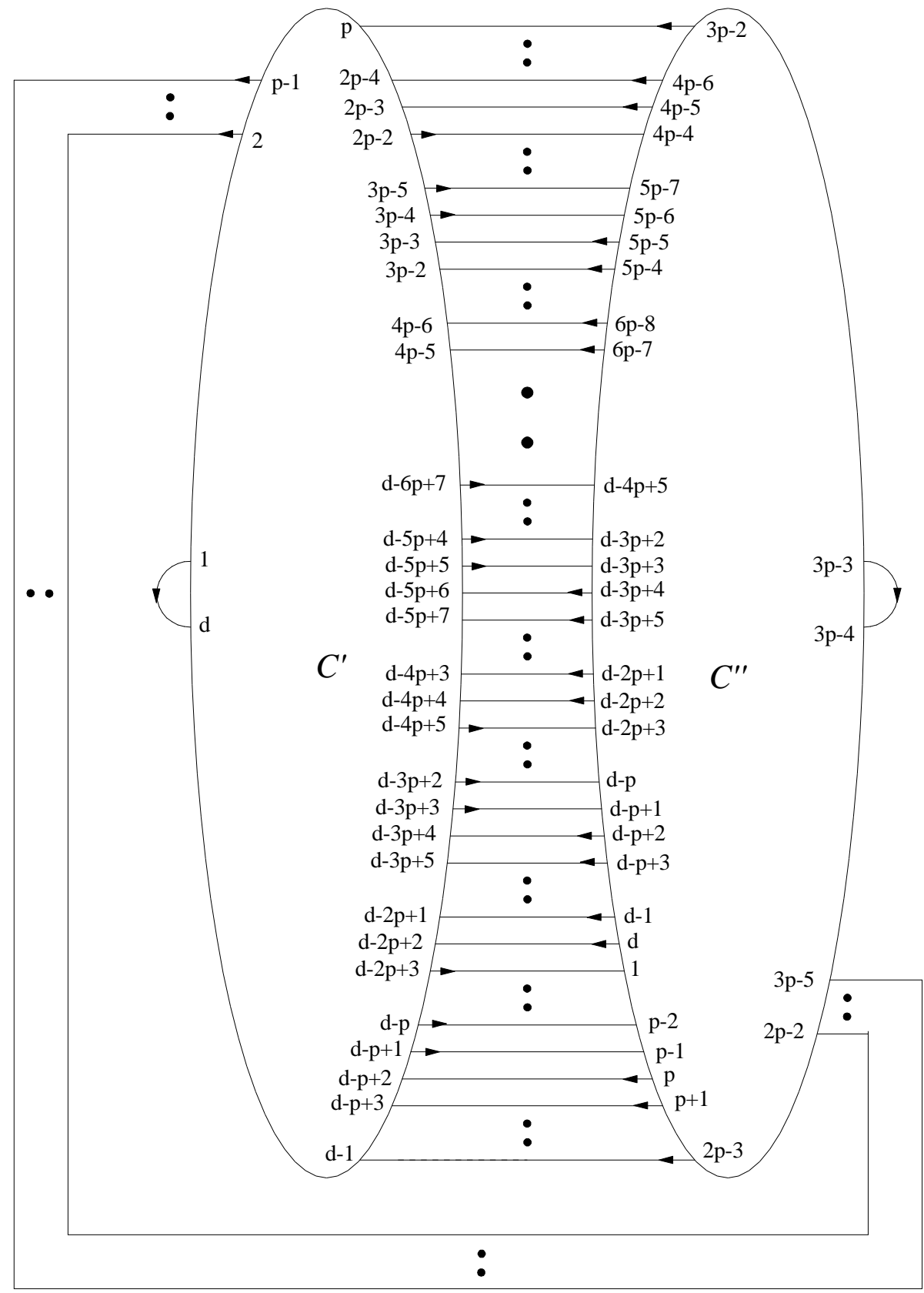

Figure 7: $D(1, p-2,2 m p-2 m-p-1,1,-3 p+4,0)$. 
Acknowledgements. Work partially developed during a visit by the third named author to the Department of Mathematics of Atatürk University, Erzurum (Turkey), supported by TOKTEN/UNISTAR funds, and performed under the auspices of the G.N.S.A.G.A. of I.N.d.A.M. (Italy) and the University of Bologna funds for selected research topics.

\section{References}

[1] A. Cattabriga and M. Mulazzani: Strongly-cyclic branched coverings of $(1,1)$-knots and cyclic presentation of groups. Math. Proc. Camb. Philos. Soc. (2003), to appear, arXiv:math.GT/0110042.

[2] A. Cattabriga and M. Mulazzani: (1,1)-knots via the mapping class group of the twice punctured torus. Adv. Geom. (2003), to appear, arXiv:math.GT/0205138

[3] A. Cattabriga and M. Mulazzani: All strongly-cyclic branched coverings of (1,1)-knots are Dunwoody manifolds. Preprint (2003).

[4] A. Cavicchioli, F. Hegenbarth and A. C. Kim: A geometric study of Sieradsky groups. Algebra Colloq. 5 (1998), 203-217.

[5] M. J. Dunwoody: Cyclic presentations and 3-manifolds. In: Proc. Inter. Conf., Groups-Korea '94, Walter de Gruyter, Berlin-New York (1995), $47-55$.

[6] L. Grasselli and M. Mulazzani: Genus one 1-bridge knots and Dunwoody manifolds. Forum Math. 13 (2001), 379-397.

[7] H. Helling, A. C. Kim and J. L. Mennicke: A geometric study of Fibonacci groups. J. Lie Theory 8 (1998), 1-23.

[8] D. L. Johnson, Topics in the theory of group presentations. London Math. Soc. Lect. Note Ser. 42, Cambridge Univ. Press, 1980.

[9] A. Kawauchi: A survey on knot theory. Birkhäusen Verlag, Basel, 1996.

[10] A. C. Kim: On the Fibonacci group and related topics. Contemp. Math. 184 (1995), 231-235. 
[11] A. C. Kim, Y. Kim and A. Vesnin: On a class of cyclically presented groups. In: Proc. Inter. Conf., Groups-Korea '98, Walter de Gruyter, Berlin-New York (2000), 211-220.

[12] C. Maclachlan and A. W. Reid: Generalised Fibonacci manifolds. Transform. Groups 2 (1997), 165-182.

[13] M. Mulazzani: Cyclic presentations of groups and cyclic branched coverings of (1,1)-knots. Bull. Korean Math. Soc. 40 (2003), 101-108.

[14] F.H. Norwood: Every two-generator knot is prime. Proc. Amer. Math. Soc. 86 (1982) 143-147.

[15] D. Rolfsen: Knots and links. Publish or Perish, Berkeley, 1976.

[16] A. Vesnin and A. C. Kim: The fractional Fibonacci groups and manifolds. Sib. Math. J. 39 (1998), 655-664.

HÜSEYIN AYDIN, Atatürk University, Faculty of Art and Sciences, Department of Mathematics, Erzurum, Turkey. E-mail: aydinh@atauni.edu.tr

INCI GÜLTEKYN, Atatürk University, Faculty of Art and Sciences, Department of Mathematics, Erzurum, Turkey. E-mail: inciakarg@yahoo.com

MICHELE MULAZZANI, Department of Mathematics and C.I.R.A.M., University of Bologna, Bologna, Italy. E-mail: mulazza@dm.unibo.it 